Journal of

Case Reports: Open Access

\title{
Ovotesticular Mixed Germ Cell Tumor in a 46, XY/46, XX Hermaphrodite: A Case Report
}

\section{Roizza Beth So ${ }^{1,}$, Orolfo-Real Irisyl ${ }^{1}$, Toral Jean Anne ${ }^{2}$, Paloyo Siegfredo ${ }^{3}$}

${ }^{1}$ Section of Medical Oncology, Department of Medicine,University of the Philippines - Philippine General Hospital, Manila, Philippines

${ }^{2}$ Department of Obstetrics and Gynecology, University of the Philippines - Philippine General Hospital, Manila, Philippines ${ }^{3}$ Department of Surgery, University of the Philippines - Philippine General Hospital, Manila, Philippines

${ }^{*}$ Corresponding author: Roizza Beth So, Section of Medical Oncology, Department of Medicine,University of the Philippines

- Philippine General Hospital, Manila, Philippines; E-mail: roizza@yahoo.com

Received Date: July 29, 2019 Accepted Date: August 25, 2019 Published Date: August 28, 2019

Citation: Roizza Beth So (2019) Ovotesticular Mixed Germ Cell Tumor in a 46, XY/46, XX Hermaphrodite: A Case Report. Case Reports: Open Access 4: 1-9.

\begin{abstract}
We present a 27-year-old true hermaphrodite born with ambiguous genitalia, diagnosed to have an ovotesticular disorder of sexual development (OT-DSD) 46, XY/46, XX karyotype as a young adult. He presented with a 2-month history of an abdominal mass, accompanied by abdominal pain and narrow stools. Work-up was suspicious for a germ cell tumor, hence he underwent exploratory laparotomy, total abdominal hysterectomy, bilateral salphingo-oophorectomy, and bilateral lymph node dissection. Histopathology revealed a left ovarian mixed germ cell tumor: yolk sac tumor (60\%) and immature teratoma WHO grade 2 (40\%), and right testicular gonadoblastoma (40\%) with seminoma (60\%). Adjuvant treatment with bleomycin, etoposide, cisplatin for 2 cycles was administered and serial monitoring showed decreasing levels of serum beta-human chorionic gonadotropin and alpha-fetoprotein; however, new cervical and retroperitoneal masses developed. The patient declined surgery, hence second-line combination chemotherapy using paclitaxel, ifosfamide, and cisplatin was started. Due to acute kidney injury and new-onset neurologic symptoms while on treatment, the patient refused further work-up and treatment. Presently, he is on best supportive care.

Ovotesticular disorder of sexual development is the rarest disorder of sexual differentiation. It requires the presence of both follicle-containing ovarian tissue and testicular tissue in an individual. Dysgenetic gonads may increase the risk for malignant transformation in 3-5\% of cases. We discuss the genetics, gender assignment, pathophysiology, diagnostics, therapeutics and prognosis of such patients. A multi-disciplinary approach is emphasized for these individuals.
\end{abstract}

Keywords: 46, XY/46, XX; disorders of sex development; germ cell tumors.

(C)2019 The Authors. Published by the JScholar under the terms of the Creative Commons Attribution License http://creativecommons.org/licenses/ by/3.0/, which permits unrestricted use, provided the original author and source are credited. 


\section{Introduction}

Disorders of sexual development pertain to discordant genetic and phenotypic sex due to disordered development of the genitalia and gonads. Ovotesticular disorder of sexual development (OT-DSD) or true hermaphroditism is the rarest disorder of sexual differentiation, with an approximate of 500 reported cases worldwide and comprises less than $10 \%$ of all DSD [1]. The phenotypic spectrum may vary from normal male or female external genitalia to various degrees of ambiguity. The most common karyotype is $46, \mathrm{XX}$, followed by $46, \mathrm{XX} / 46, \mathrm{XY}$ chimerism or mosaicism, and 46,XY [2]. Malignant transformation of dysgenetic gonads increases with the presence of the $\mathrm{Y}$ chromosome and has an estimated frequency of 3-5\% [1,2]. Germ cell tumors (GCT) arise from primordial cells of the testes and ovaries and account for $5 \%$ of ovarian cancers and $95 \%$ of testicular cancers.

Diagnosis and management of OT-DSDvaries depending on the age of diagnosis, external and internal genital development, and reproductive capacity. True hermaphrodites also uniquely tackle ethical issues of gender assignment, reproductive, and psychosocial issues. Ovotesticular GCT warrants a multidisciplinary approach due to its rarity and complexity. The purpose of this paper is to present a case of an ovotesticular mixed GCT and to highlight its pathophysiology, diagnosis, treatment, and prognosis.

\section{Case}

Our patient is a true hermaphrodite, born full-term via spontaneous vaginal delivery,from a 29-year-old G4P1 (1020) mother with unremarkable prenatal history and non-consanguineous marriage. The patient was noted to have ambiguous genitalia at birth but was raised as a female per mother's decision. No further work-up was done. Developmental milestones, growth stature, and intelligence were normal for age. The patient was reared as a female and pubarche and thelarche occurred at 13 years old. However, delayed pubertal changes were observed and the patient's gender preference shifted to being a male attracted to females. Due to primary amenorrhea and features of masculinization, they sought genetic consult, and chromosomal analysis revealed a mosaic 46, XX/46, XY karyotype when he was age 21 .

Six years later, at the age of 27 , he sought to consult at our institution due to a two-month history of progressive hypogastric pain associated with a palpable abdominal mass, constipation, and narrow stools. Pertinent physical examination findings included non-dysmorphic facial features, absence of facial hair or acne, female pattern of axillary hair, tanner stage IV of the breast (Figure 1), presence of a palpable, non-tender abdominal mass, tanner stage IV of the pubic hair (Figure 2), ambiguous genitalia described as presence of a $4 \mathrm{~cm}$ infantile phallus,scrutinization of the labia majora, presence of a labia minora, urethra and vagina, and an anogenital ratio of $>0.5$ suggestive of posterior labial fusion (Figure 3 and 4).

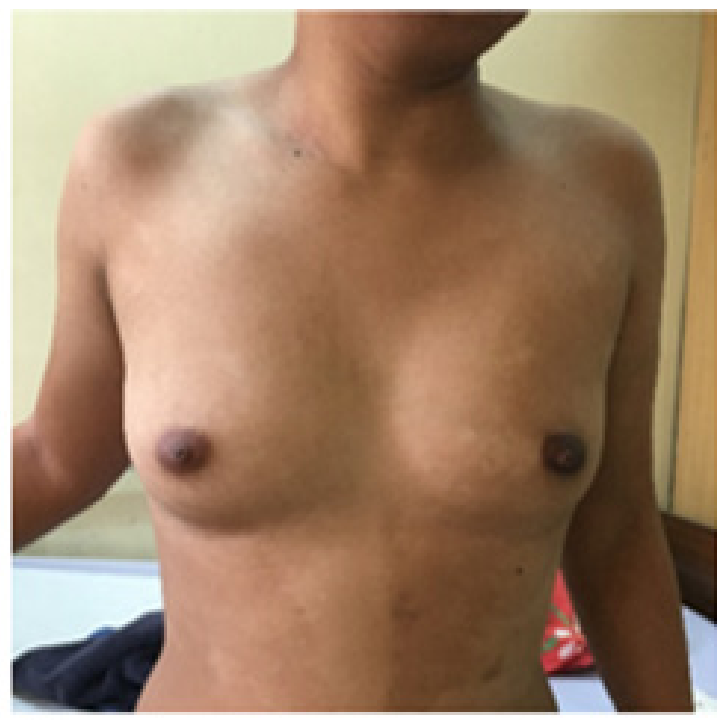

Figure 1: Tanner stage IV breast

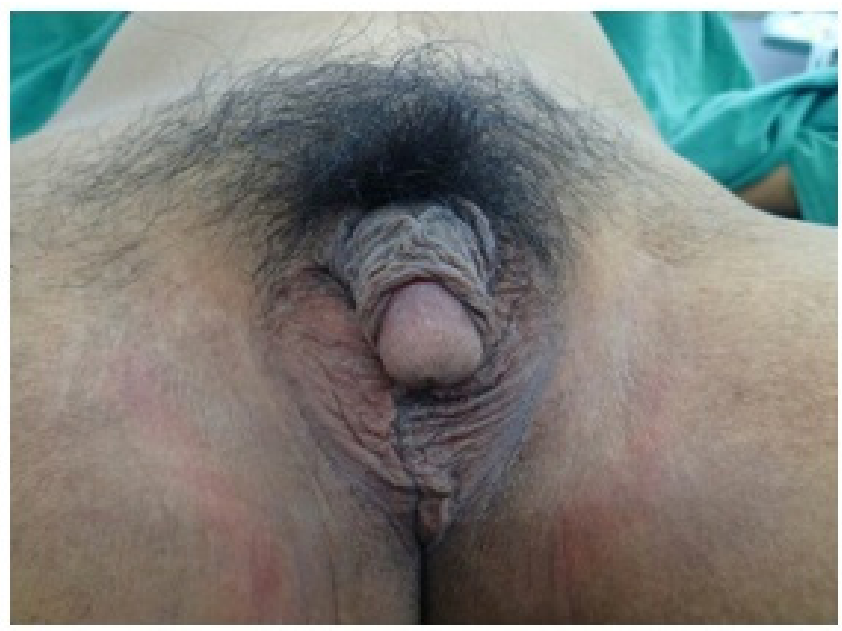

Figure 2: Tanner stage IV pubic hair, Tanner stage III male genitalia, and bilateral undescended testes 


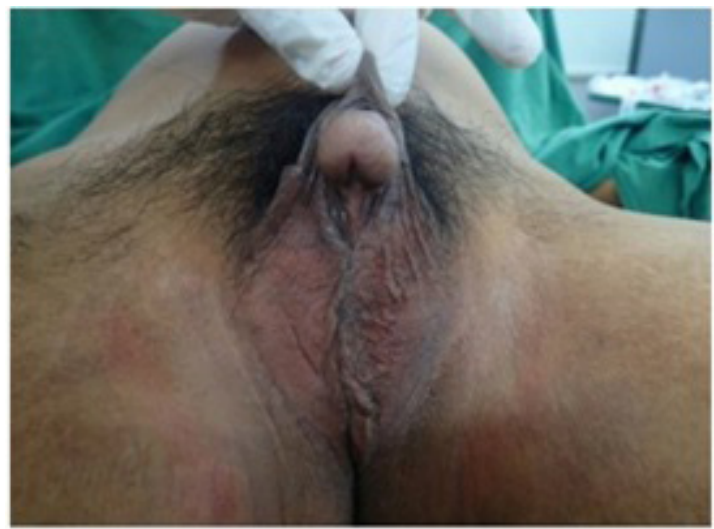

Figure 3: Micropenis without urethral opening, and scrotalization of labia majora

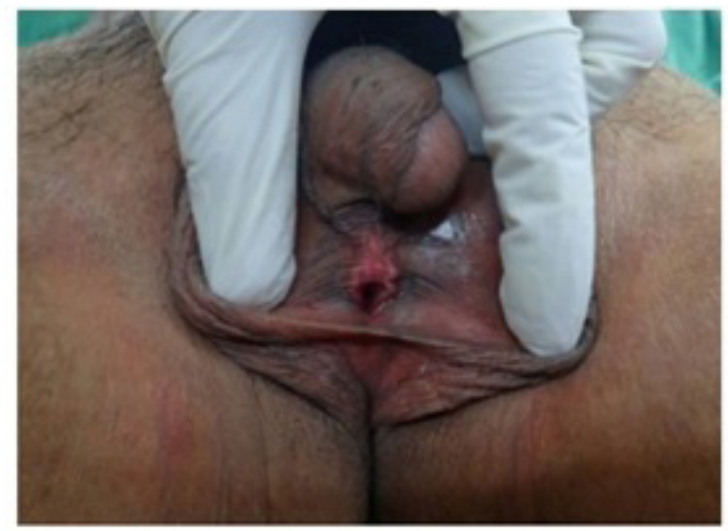

Figure 4: Ambiguous external genitalia, presence of phallus and vagina, with an anogenital ratio of $>0.5$ suggestive of posterior labial fusion
Abdominopelvic CT scan with triple contrast revealed a left abdominopelvic mass measuring $20 \times 14$ x $10.6 \mathrm{~cm}$ with retroperitoneal lymphadenopathies. Meanwhile, genitalia findings included an empty scrotum composed of fatty tissue representing the labia, a tubular focus in the perineal region representing either a prominent clitoris or an infantile penis, presence of a uterus, and non-visualization of the testes and prostate gland.Baseline alpha-fetoprotein (AFP) was $>1660$ $\mathrm{IU} / \mathrm{mL}$ (normal range 0.74-7.3), beta-human chorionic gonadotropin $(\beta-\mathrm{HCG})$ was $565 \mathrm{mIU} / \mathrm{mL}$ (normal range $<5$ ) and lactate dehydrogenase (LDH)was 2838 IU/L (normal range 313618). Metastatic workup and blood chemistries were normal. The patient underwent exploratory laparotomy, peritoneal fluid cytology, omentectomy, total abdominal hysterectomy, bilateral scalping-oophorectomy, and bilateral lymph node dissection. Intra-operative findings revealed a smooth peritoneal surface, a left ovarian solid mass measuring $21 \times 14 \times 8 \mathrm{~cm}$, and a grossly normal right ovary. Cut section revealed necrotic areas suspicious for malignancies and an infantile uterus. (Figures 5 to 11 )
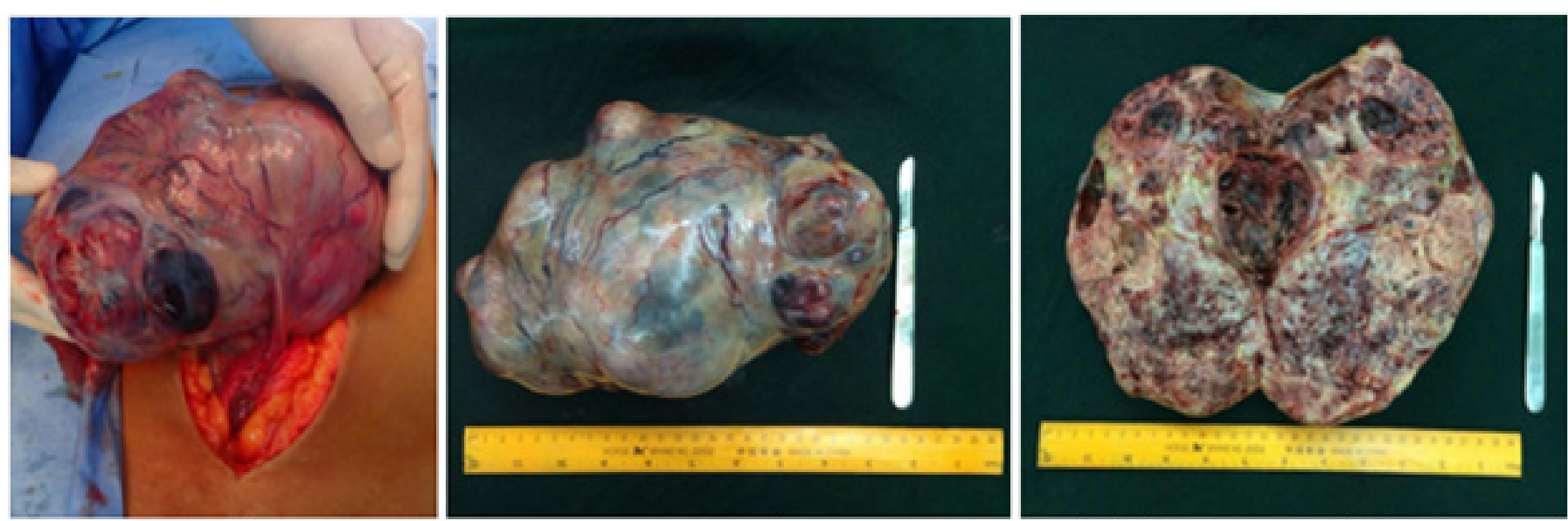

Figure 5, 6, 7: Left ovary- $21 \times 14 \times 8 \mathrm{~cm}$ gray-tan ovoid mass, cut section of the left ovary revealed a cream tan to yellow gray variegated, solid, fleshy surface; with friable necrotic material. 

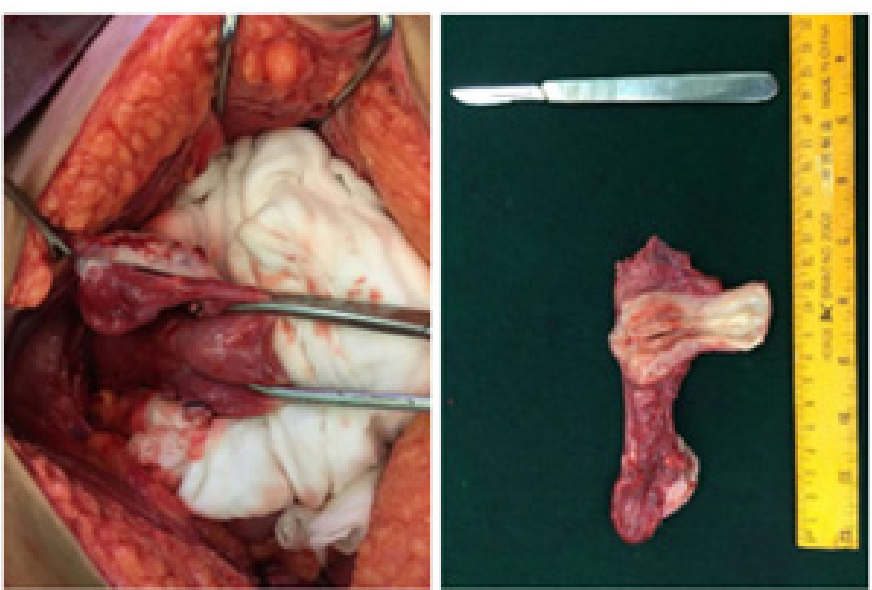

Figure 8-9: Uterus with right adnexa: $7 \times 3 \times 3 \mathrm{~cm}$ pink-tan uterus, with a uterine canal of $6 \mathrm{~cm}$; cervix of $1.5 \times 1.5 \times 1.5 \mathrm{~cm}$ with a cream-white smooth ectocervix and light cream endocervical canal.
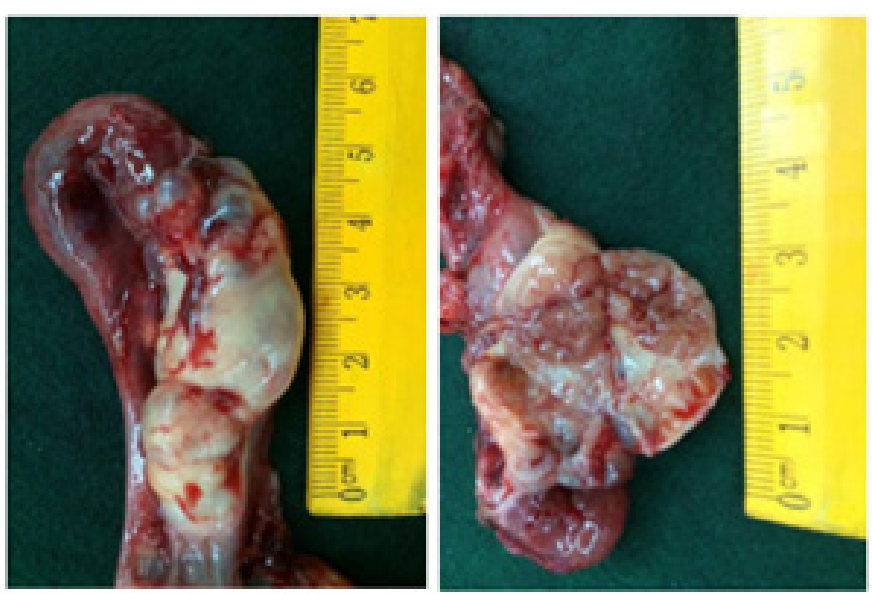

Figure 10-11: Right adnexa: $2.5 \times 1.8 \times 1.5 \mathrm{~cm}$ creamyellow ovary, fallopian tube $6 \mathrm{~cm}$ long with a $1 \mathrm{~cm}$ diameter, cut sections of the ovary reveal an intraparenchymal solid cream-yellow to brown ovoid mass measuring $1.1 \times 1 \times 0.8 \mathrm{~cm}$.

Histopathology of the left ovary and fallopian tube revealed a mixed germ cell tumor comprised of a yolk sac tumor (60\%, Figure 12-13) and immature teratoma WHO grade 2 (40\%, Figure 14-15).

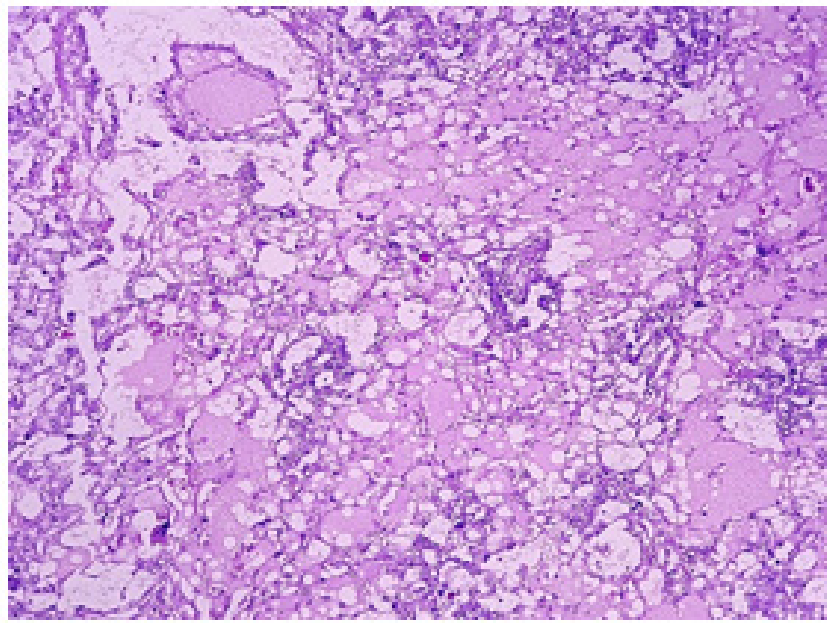

Figure 12 (100X): Primitive-appearing tumor cells admixed with microcystic spaces containing eosinophilic material. Eosinophilic, hyaline globules are also present.

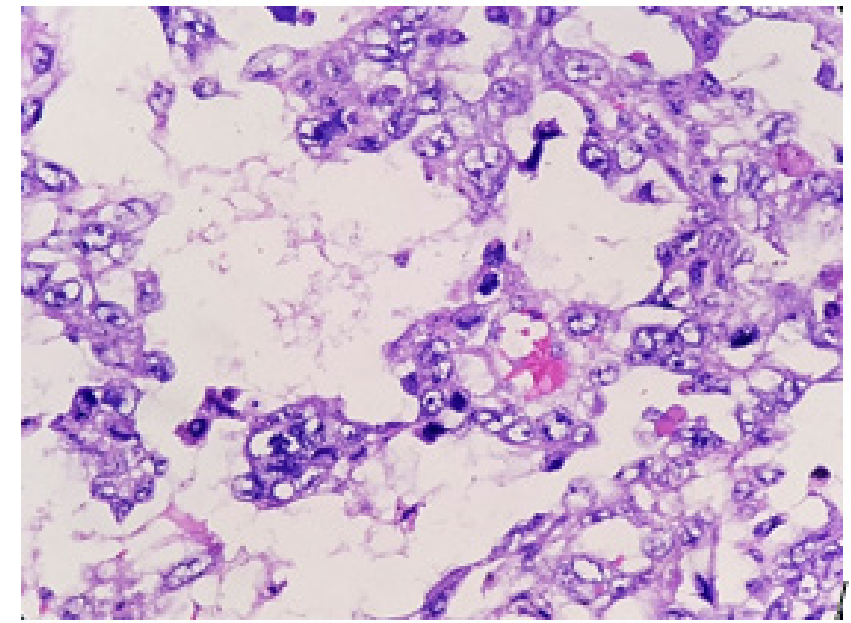

Figure 13 (400X): Pleomorphic tumor cells with primitive-looking, vesicular and irregular nuclei and prominent nucleoli. Schiller-duval body is seen. Features consistent with a yolk sac tumor. 


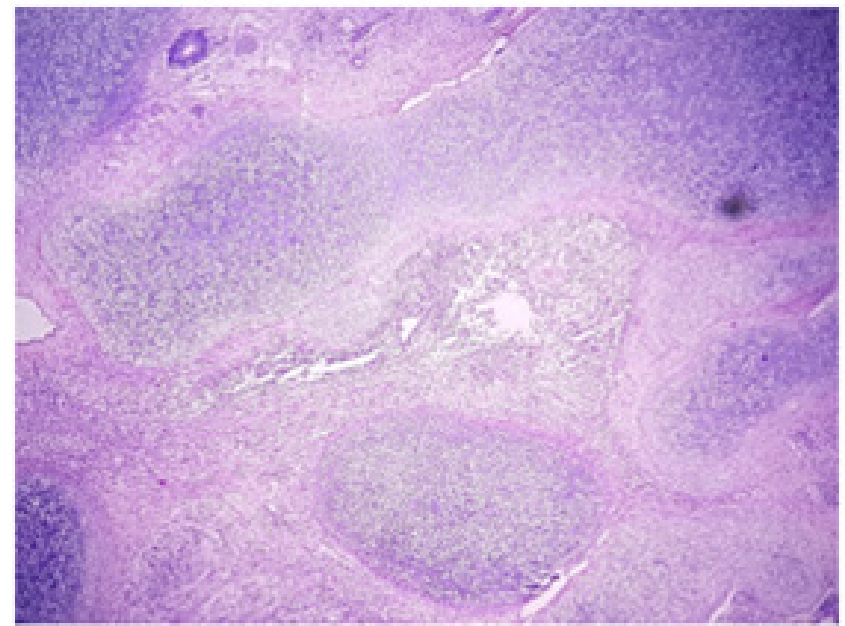

Figure 14 (40X): An area showing wide areas of immature cartilage and connective tissue.

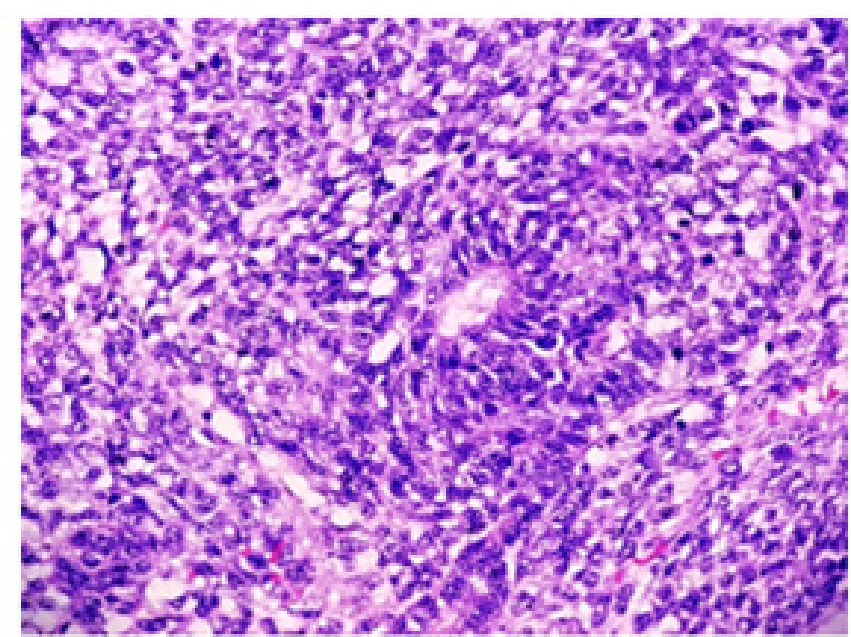

Figure 15 (400X): A focus of immature neuroepithelium with a true rosette; morphologically compatible with an immature teratoma

The right adnexa (Figure 16-17)revealed the presence of a gonadoblastoma (40\%, Figure 18) and seminoma (60\%, Figure 19). The uterus showed a disordered proliferative endometrium and the cervix had squamous metaplasia. Histopathology was negative for tumors in the right ovarian, fallopian tube, epididymis and seminal vesicle. The bilateral pelvic lymph nodes, omentum, and peritoneal fluid cytology were likewise negative for malignant cells.

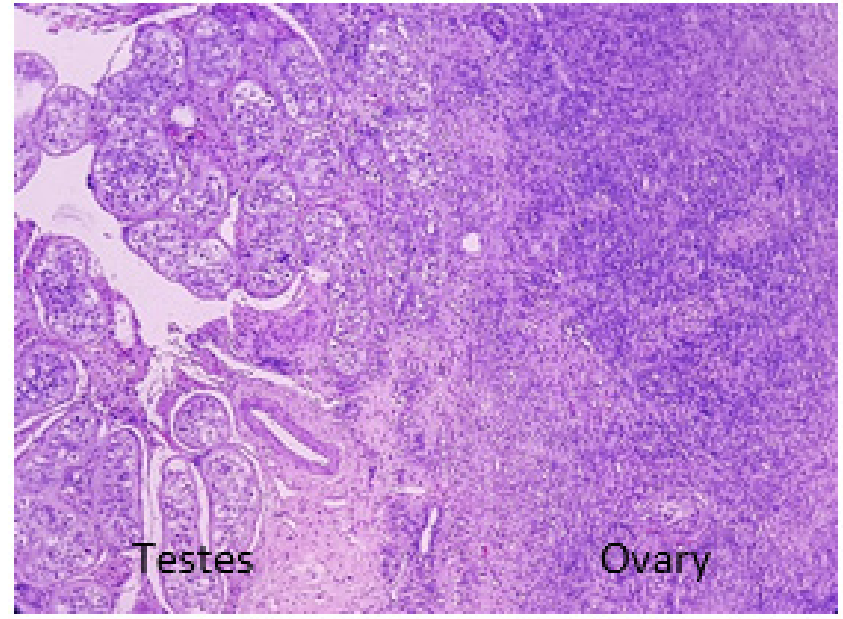

Figure 16 (100X): Normal testicular tissues on the left showing atrophic changes admixed with ovarian stromal tissues on the right.

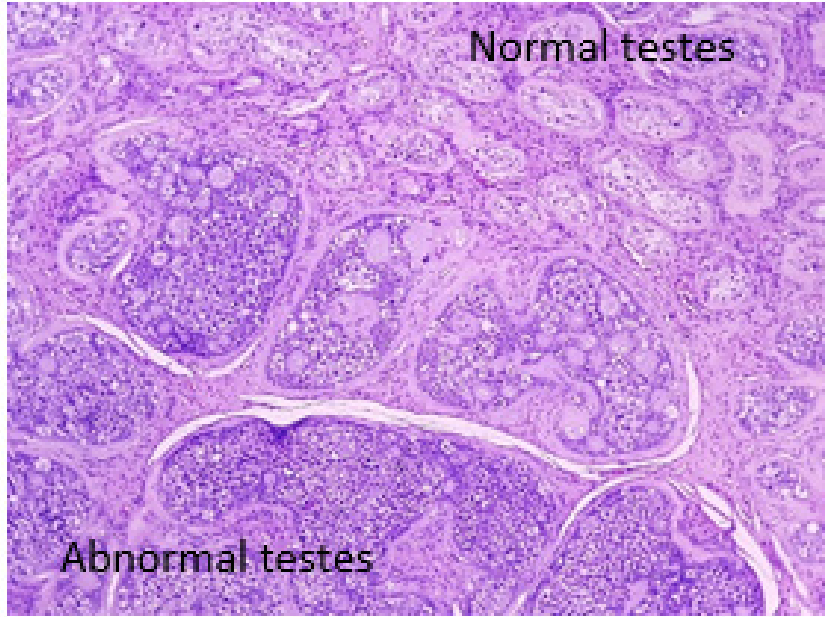

Figure 17 (100X): Tumor nests (lower half) seen encroaching on normal testicular tissues (upper right). 


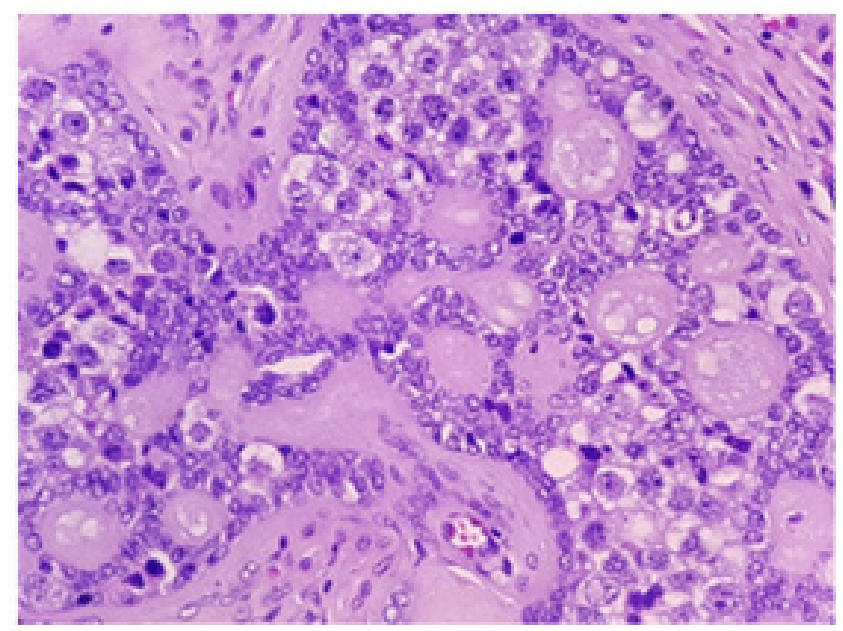

Figure 18 (400X): The tumor contains a dual population of cells - germ cells and sex cord stromal cells - along with round spaces containing eosinophilic material. Morphologically compatible with a gonadoblastoma.

Repeat tumor markers postoperatively showed decreased LDH at $763 \mathrm{IU} / \mathrm{L}$, but the further elevation of beta-HCG at $815 \mathrm{mIU} / \mathrm{mL}$ and AFP at $>1660 \mathrm{IU} / \mathrm{mL}$. (Table 1) Patient was assessed to have ovarian mixed germ cell tumor (yolk sac tumor 60\%, immature teratoma WHO grade $240 \%$ ); right:pT1a pN0 cM0, TNM stage 1A, FIGO stage 1, and testicular seminoma (60\%); left:pT1 pN0 cM0 S2, stage IS; and gonadoblastoma (40\%). The patient was advised adjuvant chemotherapy. One-month postoperative physical examination revealed the presence of a new left paracervical lymphadenopathy measuring $4 \times 5 \mathrm{~cm}$. The patient was advised to repeat metastatic workup and biopsy of the cervical mass however was not done due to financial constraints. The patient underwent chemotherapy with bleomycin, etoposide, cisplatin (BEP) regimen. After 2 cycles, serial monitoring of tumor markers showed a significant decrease of AFP to $1044 \mathrm{IU} / \mathrm{mL}$ and beta-HCG at $6.32 \mathrm{mIU} / \mathrm{mL}$. (Table 1) However, he developed moderate dyspnea, pleuritic chest pain, and enlargement of the left paracervical mass.

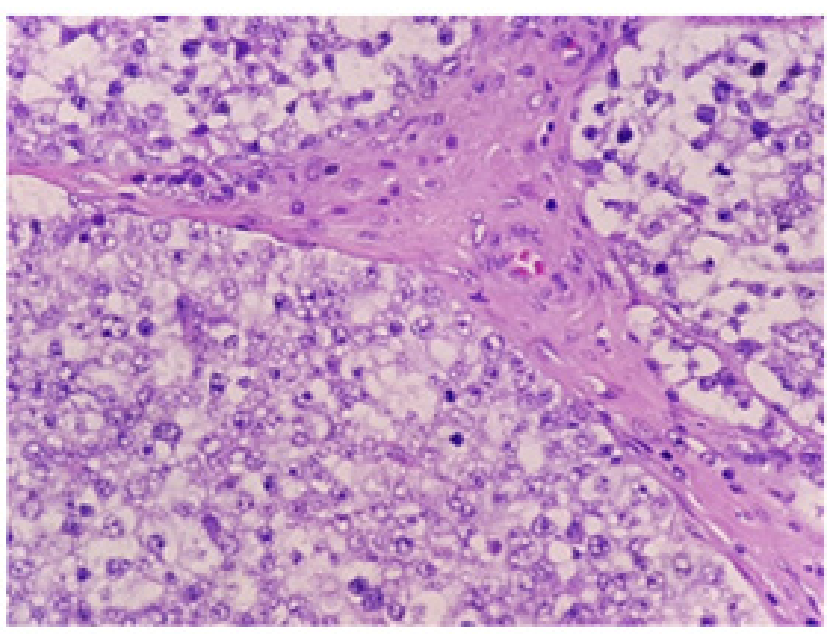

Figure 19 (400X) Well-defined tumor nests composed of germ cells with clear cytoplasm and primitive nuclei. Morphologically compatible with a seminoma.

The patient consented for fine-needle aspiration biopsy (FNAB) of the enlarging mass which revealed atypical cells positive for malignancy. Repeat cervical and chest CT scan showed a left cystic supraclavicular mass, pleural based nodules likely metastases, and confluent left paravertebral lymphadenopathies. Repeat abdominal CT scan revealed a new retroperitoneal mass measuring 14.7 x 12.3 x 9.2cmwith bilateral obstructive nephropathy. Due to disease progression, chemotherapy was shifted to paclitaxel, ifosfamide, and cisplatin (TIP) regimen every 3 weeks and the patient was able to tolerate 3 cycles without significant adverse reactions and complications. Repeat tumor markers showed a further decrease of AFP at $75 \mathrm{IU} / \mathrm{mL}$ and beta-HCG at $1.21 \mathrm{mIU} / \mathrm{mL}$. (Table 1 ) However, after the $3^{\text {rd }}$ cycle of TIP, he developed neurologic symptoms characterized by incoherent speech and focal intermittent jerking of bilateral lower extremities. He also developed acute kidney injury accompanied by hypokalemia and hypomagnesemia. The patient was advised cranial MRI however he refused further work-up and treatment.

\begin{tabular}{|l|l|l|l|l|l|l|}
\hline $\begin{array}{l}\text { Tumor } \\
\text { Marker }\end{array}$ & Normal Values & $\begin{array}{l}\text { Pre-opera- } \\
\text { tive }\end{array}$ & $\begin{array}{l}\text { Post-oper- } \\
\text { ative }\end{array}$ & $\begin{array}{l}\text { After 2 } \\
\text { cycle BEP }\end{array}$ & $\begin{array}{l}\text { After 2 } \\
\text { cycle of TIP }\end{array}$ & $\begin{array}{l}5 \text { months on best } \\
\text { supportive care }\end{array}$ \\
\hline AFP & $0.74-7.3 \mathrm{IU} / \mathrm{mL}$ & $>1660$ & $>1660$ & 1044 & 75 & $>400$ \\
\hline B-HCG & $\begin{array}{l}<5 \mathrm{mIU} / \mathrm{mL} ; \\
\text { non-pregnant }\end{array}$ & 565 & 815 & 6.32 & 1.21 & 0 \\
\hline LDH & $313-618 \mathrm{IU} / \mathrm{L}$ & 2838 & 763 & - & - & 689 \\
\hline
\end{tabular}

Table 1. Summary of serum tumor markers 
One month after discharge, the patient was admitted for uremia which was treated as a case of acute kidney injury secondary to obstructive uropathy and cisplatin nephropathy; he underwent bilateral DJ stent insertion. He is on best supportive care and follows up for symptomatic treatment of discomfort and aspiration of the cystic cervical mass as needed.

\section{Discussion}

Ambiguous genitalia is a disorder of sexual development wherein the genital phenotype is neither male nor female. It constitutes a major social dilemma due to the distressing nature of gender allocation and is considered a medical emergency for pediatricians. True hermaphroditism is an ovotesticular disorder of sexual development (OT-DSD) that requires the presence of follicle containing ovarian tissue and testicular tissue in the same or opposite gonad [1]. It is sub-classified according to the type and location of the gonads, wherein $20 \%$ are lateral cases that have a testis on one side and an ovary on the other side, while $30 \%$ have bilateral ovotestes usually juxtaposed end-to-end, and $50 \%$ have unilateral ovotestis and an ovary or testis contralaterally [1]. Our patient has histologically proven bilateral ovotestes after removal of his diseased gonads. Diagnostic work-up for ambiguous genitalia comprises a complex network of physical examination of the external genitalia, chromosomal analysis, pelvic imaging studies or mini-laparoscopy for assessment of internal genitalia, and examination of gonadal hormones.

Karyotyping is the analysis of chromosomes obtained from tissue samples, blood, amniotic fluid, or bone marrow to detect genetic aberrations. OT-DSD can result from sex chromosome mosaicism, chimerism, or Y chromosomal translocation [2]. The most common karyotype for OT-DSD is $46, \mathrm{XX}(71 \%)$, followed by 46, XX/46, XY chimerism or mosaicism (20\%), and 46, XY (7\%) [2,8]. Individuals with a 46, XY/46, XX karyotype may either result from mosaicism or chimerism. Mosaicism results from a mitotic error, usually a nondisjunction, during the first blastomeric division or at a later stage within a single zygote. It contains two populations of genetically distinct cells in an individual who was formed from a single fertilized egg [3]. In contrast to mosaicism, chimerism results from fertilization of two oocytes by two sperms and subsequent fusion of two different zygotes into a single embryo. The karyotype of our patient obtained via blood specimen at the age of 21 years old is mosaic 46, XY/46, XX [4].

The sex assignment and treatment of true hermaphrodites depend on the age at diagnosis, genital development, internal structures, and reproductive capacity. Ideally, patients should be referred to during the neonatal period. The 46, XX karyotype is the most common karyotype for OT-DSD. These individuals are reared as females since they have functional ovaries and uterus, and the removal of testicular tissue is advised before puberty [1]. For the remaining 46, XY/46, XX and 46, XY karyotypes, a male gender can be considered if there is adequate phallic size, surgically correctable hypospadias, and sufficient testicular tissue that can descend into the scrotum; however, the majority will have azoospermia [5]. If a uterus, vagina and sufficient ovarian function is present, the child can be reared as a female. Removal of the discordant gonad is encouraged before puberty [1]. A total of 8 successful pregnancies have been reported for those who were reared as females, and a few male hermaphrodites were reported to have successfully fathered a child $[1,2,6,7]$. They also have normal life expectancies and normal intelligence.

If a patient with OT-DSD presents in late childhood or adolescence, gender identity is more complicated and is associated with more psychological distress to the child. Androgenization in girls with testicular tissue occurs and results in deepening of the voice and clitoral enlargement. Estrogenization in boys with ovarian tissue may also occur, and results to gynecomastia and occasionally menstruation manifesting as hematuria [1-2]. After appropriate workup and counseling, the discordant gonad should be removed and sex hormone supplementation may be required for proper pubertal development. Our patient only sought medical consult at the age of 21 and he was already suffering from gender dysphoria. His pubertal changes of both estrogenization and androgenization can be both explained due to the presence of ovotesticular function. The prevalence of testicular gonadoblastoma or germinoma is approximately 3-5\% and further increases if cryptorchidism is present; hence, removal of the dysgenetic gonad is highly recommended [1-2].

Germ cell tumors are derived from the primordial germ cells of the ovary and testis. Due to its rarity, the prevalence of ovotesticular malignant mixed germ cell tumors is unknown. Ovarian germ cell tumors (OGCT) usually present with abdominal enlargement or abdominal pain, while testicular germ cell tumors usually present as a painless unilateral testicular mass or as a dull ache in the lower abdomen. High resolution abdominopelvic computed tomography (CT) scan with contrast, a chest radiograph, and serum tumor markers ( $\beta$-HCG, AFP and $\mathrm{LDH})$ are required for proper assessment. Serum tumor markers should be obtained pre-treatment, then repeated post-operatively for staging completion. Serial monitoring of tumor markers is essential for detection of residual disease and response to treatment. Serum half-lives of $\beta$-HCG (1.5-3 days) and AFP (5-7 
days) are important considerations during monitoring of response to treatment [9].

Gonadoblastomas contain germ cells and stromal cells arising from a dysgenetic testis, and overgrowth of the germinal components leads to the formation of seminoma in $50 \%$ of the cases. Since the patient's right testes showed gonadoblastoma (40\%) and seminoma (60\%) but have elevated AFP post-orchiectomy, we treated the patient as a case of nonseminomatous germ cell tumor (NSGCT). Stage IS (abnormal tumor markers without lymph node metastases) are treated the same as stage III because of the assumption of occult metastatic disease. Malignant ovarian GCT consists of two or more admixed types of germ cell tumors. In our case, histopathology revealed yolk sac tumor (60\%) and immature teratoma grade 2 (40\%). Yolk sac tumors growth can be very rapid and aggressive with extensive intraperitoneal dissemination. Immature teratomas are graded depending on the amount of immature neural elements (ranging from grade 1 - well-differentiated to grade 3 - poorly differentiated), and grade is an important risk indicator for extra ovarian spread. The presence of yolk sac tumor in immature teratomas generally reflects a more aggressive behavior and a worse outcome [10].

Surgery is essential for diagnosis, staging, and treatment. Incorporations of platinum agents in both testicular and ovarian GCT is the current standard of care as adjuvant treatment. Cisplatin-based combination chemotherapy can cure patients even with disseminated GCTs [11]. Current category 1 adjuvant treatment regimen for both testicular and ovarian GCT is bleomycin, etoposide, cisplatin (BEP). Assessment of serial tumor markers ( $\beta$-HCG and AFP) is important to monitor response to treatment wherein decreasing values represent an adequate tumor response; while a plateau or rise in marker levels indicate relapse or resistance to therapy. Typically, a good prognosis is observed in $90 \%$ of women with early-stage OGCTs, with up to $80 \%$ long term survivors. Good-risk NSGCTs have at least $80 \%$ relapse-free survival; however, up to $60 \%$ of intermediate or poor-risk NSGCTs relapse and require additional treatment [11]. Our patient had decreasing tumor marker levels after BEP but due to clinical progression, the regimen was shifted to paclitaxel, ifosfamide, cisplatin (TIP). The patient was advised regarding surgical removal of residual disease; however, he did not consent. Patients with NSGCT may be complicated by the presence of a metastatic teratoma component which is insensitive to chemotherapy, and surgery is the only curative management. A growing teratoma should be considered when a mass grows during chemotherapy especially when serum tumor markers are declining [11].

\section{Summary}

Early detection and referral of a patient with ambiguous genitalia can provide improved quality of life, and prevent gender dysphoria and psychosocial dilemmas. Prophylactic removal of the dysgenetic gonad may prevent risk for malignant transformation. Patients with OT-DSDs have normal life spans, normal intelligence, and may have reproductive potentials. Ovotesticular mixed GCT requires individualized holistic treatment plans and entails a multidisciplinary approach which should include psychosocial support for optimal outcomes. 


\section{Reference}

1. Achermann J, Hughes I (2016) Pediatric disorders of sex development. Section V reproduction. Williams Textbook of endocrinology. Thirteenth edition.

2. Kolon T, et al. (2007) Disorders of sexual development. Textbook of penn clinical manual of urology.

3. Raby B, et al. (2018) Inheritance patterns of monogenic disorders (mendelian and non-mendelian).

4. Malan V and Gesny R (2007) Prenatal diagnosis and normal outcome of a 46, XX/46, XY chimera: a case report. Human reproduction 22: 1037-1041.

5. Bouvattier C (2010) Bisexual gonads: true hermaphroditism. Disorders of sex development. Textbook of pediatric urology.

6. Talerman A, et al (1990) True hermaphrodite with bilateral ovotestes, bilateral gonadoblastomas and dysgerminomas, 46, XX/46, XY karyotype, and successful pregnancy. Cancer 66: $2668-2672$.

7. Arboleda V, et al. (2014) Disorders of sex development. Yen and Jaffe's reproductive endocrinology. Seventh edition.

8. Krob G, Kuhnle U, et al. (1994) True hermaphroditism: geographical distribution, clinical findings, chromosomes, and gonadal histology. Eur j Pediatr153: 2-10.

9. Michaelson M, Oh W, et al. (2018) Serum tumor markers in testicular germ cell tumors.

10. Hochberg L, Hoffman M, et al. (2018) Differential diagnosis of the adnexal mass.

11. Gilican T, Kantoff P, et al. (2018) Diagnosis and treatment of relapsed and refractory testicular germ cell tumors.

12. Houk C, et al. (2018) Evaluation of the infant with atypical genitalia (a disorder of sex development)

13. Biro F, Chan Y (2018) Normal puberty.

14. Hiort O (2018) Normal sexual development.
15. Hirsch M, et al. (2018) Anatomy and pathology of testicular tumors. January 2018.

16. Michaelson M, Oh K (2018) Epidemiology of and risk factors for testicular germ cell tumors.

17. Steele G, Richie J, et al. (2018) Clinical manifestations, diagnosis, and staging of testicular germ cell tumors.

18. Kantoff P, Giligan T, et al (2018) Initial risk-stratified treatment for advanced testicular germ cell tumors.

19. Oh W (2018) Overview of the treatment of testicular germ cell tumors. January 2018.

20. Gershenson D (2018) Ovarian germ cell tumors: pathology, clinical manifestations, and diagnosis.

21. Gershenson D (2018) Treatment of malignant germ cell tumors of the ovary.

Submit your manuscript to a JScholar journal and benefit from:

- Convenient online submission

व Rigorous peer review

I Immediate publication on acceptance

ब Open access: articles freely available online

ब High visibility within the field

ब Better discount for your subsequent articles

Submit your manuscript at http://www.jscholaronline.org/submit-manuscript.php 